\title{
Hierarchical network enabled flexible textile pressure sensor with ultra-broad response range and high-temperature resistance
}

\section{Meiling Jia}

Shenzhen Institutes of Advanced Technology, Chinese Academy of Sciences

\section{Chenghan Yi}

Shenzhen Institutes of Advanced Technology, Chinese Academy of Sciences

\section{Yankun Han}

Shenzhen Institutes of Advanced Technology, Chinese Academy of Sciences Xin Li

Shenzhen Institutes of Advanced Technology, Chinese Academy of Sciences

\section{Guoliang Xu}

Shenzhen Institutes of Advanced Technology, Chinese Academy of Sciences

\section{$\mathrm{Ke} \mathrm{He}$}

Shenzhen Institutes of Advanced Technology, Chinese Academy of Sciences

Nianci Li

Shenzhen Institutes of Advanced Technology, Chinese Academy of Sciences

\section{Yuxin Hou}

Shenzhen Institutes of Advanced Technology, Chinese Academy of Sciences

\section{Zhongguo Wang}

Shenzhen Institutes of Advanced Technology, Chinese Academy of Sciences

\section{Yuanhao Zhu}

Shenzhen Institutes of Advanced Technology, Chinese Academy of Sciences

\section{Yuanao Zhang}

Shenzhen Institutes of Advanced Technology, Chinese Academy of Sciences

\section{Peifei Tong}

Shenzhen Institutes of Advanced Technology, Chinese Academy of Sciences

\section{Jiawei Yang}

Shenzhen Institutes of Advanced Technology, Chinese Academy of Sciences

\section{Yougen Hu}

Shenzhen Institutes of Advanced Technology, Chinese Academy of Sciences

\section{Zhixun Wang}


School of Electrical and Electronic Engineering, Nanyang Technological University, 50 Nanyang Avenue, 639798 https://orcid.org/0000-0001-9918-9939

\section{Weimin Li}

Shenzhen Institutes of Advanced Technology, Chinese Academy of Sciences

\section{Wenjie Li}

Shenzhen Institutes of Advanced Technology

\section{Lei Wei}

School of Electrical and Electronic Engineering, Nanyang Technological University https://orcid.org/0000-0003-0819-8325

\section{Chunlei Yang}

Chinese Academy of Sciences

\section{Ming Chen ( $\nabla$ ming.chen2@siat.ac.cn )}

Shenzhen Institutes of Advanced Technology, Chinese Academy of Sciences

\section{Research Article}

Keywords: pressure sensors, polyimide fabric, sensitivity, linearity range, high pressure, high temperature Posted Date: December 8th, 2021

DOI: https://doi.org/10.21203/rs.3.rs-633054/v2

License: (c) (1) This work is licensed under a Creative Commons Attribution 4.0 International License. Read Full License 


\section{Abstract}

Thin, lightweight, and flexible textile pressure sensors with the ability to detect the full range of faint pressure ( $<100 \mathrm{~Pa}$ ), low pressure (in the range of $\mathrm{KPa}$ ) and high pressure (in the range of $\mathrm{MPa}$ ) are in significant demand to meet the requirements for applications in daily activities and more meaningfully in some harsh environments, such as high temperature and high pressure. However, it is still a significant challenge to fulfill these requirements simultaneously in a single pressure sensor. Herein, a highperformance pressure sensor enabled by polyimide fiber fabric with functionalized carbon-nanotube (PI/FCNT) is obtained via a facile electrophoretic deposition (EPD) approach. High-density FCNT is evenly wrapped and chemically bonded to the fiber surface during the EPD process, forming a conductive hierarchical fiber/FCNT matrix. Benefiting from the large compressible region of PI fiber fabric, abundant yet firm contacting points, point-to-point contacting mode, and high elastic modulus of both PI and CNT, the proposed PI/FCNT pressure sensor can be customized and modulated to achieve both a wide linear ranges, ultra-broad sensing range, long-term stability and high-temperature resistance. Thanks to these merits, the proposed PI/FCNT(EPD) pressure sensor could monitor the human physiological information, detect tiny and extremely high pressure, can be integrated into an intelligent mechanical hand to detect the contact force under high-temperature $\left(>300^{\circ} \mathrm{C}\right)$, endowing it with high applicability in the fields of real-time health monitoring, intelligent robots, and harsh environments.

\section{Introduction}

Thin, flexible, and wearable pressure sensors have a great application perspective in electronic skins, healthcare monitors, soft robotics, artificial intelligence, et al ${ }^{1-10}$. To date, flexible pressure sensors are mainly divided into four types: capacitive, piezoelectric, triboelectric, and piezoresistive ${ }^{11-20 .}$ Among the proposed flexible pressure sensors, piezoresistive sensors are widely used owing to their low manufacturing cost, ease of fabrication as well as easy readout mechanism. Flexible piezoresistive pressure sensors are generally comprised of three parts: flexible matrix material (such as the frequentlyused polydimethylsiloxane (PDMS), polyurethane (PU)), conductive materials (such as Au, Ag, Graphene, $\mathrm{CNT}$ ), and electrode materials.

With the change of practical applications and customer needs, in addition to fulfilling the requirements of daily life (such as wearable healthcare monitoring at normal pressure and room temperature), there is an increasing demand for wearable pressure sensors that can be used in harsh environments, such as high temperatures and/or high pressures. For instance, real-time physiological monitoring of firefighters during the firefight action is critical to assess the level of danger and make a real-time and life-saving decision to protect firefighters. Besides, the real-time detection of pre-stresses ( tens of MPa) within the composite sheath of high-speed permanent magnet motor rotor under high temperature $\left(>300^{\circ} \mathrm{C}\right)$ is vital for the whole motor system, which requires a flexible yet thin pressure sensor with the applicability in both hightemperature and high-pressure condition. Thus, there is an urgent need to achieve a single pressure 
sensor that can detect the full range of faint pressure ( $<100 \mathrm{~Pa}$ ), low-pressure (in the range of $\mathrm{KPa}$, such as human motions), high pressure (in the range of $\mathrm{MPa}$, for example, safety monitoring of road, rail, bridge, and tunnel) and can be used in harsh high-temperature and high-pressure environments. However, it is still a significant challenge.

Generally, high modulus of the sensor material is a necessary precondition to the broad sensing range and large linearity. A typical method to improve the linearity is to create nano-sized or micro-sized patterns on elastic polymer. For example, several PDMS geometrical features, including micro-pyramids, micro-hemispheres, and micro-semicylinders, are demonstrated to be an effective method to improve the linearity ${ }^{21-24}$. In our previous work ${ }^{25-28}$, we also show that the contacting modes play a substantial effect on the sensing performance and demonstrate that the point-to-point contacting mode is the optimum strategy. However, these elastic polymers are commonly subject to the disadvantage of low elastic modulus (10 KPa to $10 \mathrm{MPa}$ ) and low glass transition temperature, which reveals that the sensor response will saturate at low-pressure levels (namely narrow linearity range and sensing range), and the sensor is not suitable for the high-temperature environments. Besides, the fabrication of these micro/nano-structured elastic polymer/conductive layers often requires complex fabrication processes, such as UV lithography, ICP/RIE etching, E-beam evaporation/magnetron sputtering, et al. To address these issues, we adopt polyimide (PI) as the flexible matrix material, because PI possesses the merits of higher modulus and high-temperature-resistant properties compared to other elastic polymers ${ }^{29-30}$ (Figure 1a). However, high modulus usually leads to increased stiffness. Namely, the deformation of PI under the external pressure is not large, which may result in low sensitivity and quick signal saturation. Therefore, $\mathrm{PI}$ film with a wide compressible region is an essential prerequisite for large linearity and wide sensing range, and the appropriate design of contacting modes is crucial in order to offer large and consistent resistance change during the compressible region. Chen et al. reported a wearable pressure sensor based on $\mathrm{PI} / \mathrm{CNT}$ composite aerogels through the freeze-drying and thermal imidization process, showing a linear range of $5 \mathrm{KPa}$ and a sensing range of $61 \mathrm{KPa}$. The sensing mechanism can be attributed to the contact of the adjacent cell walls (surface contacts) upon external compression ${ }^{31}$. Jeong et al. proposed a flexible pressure sensor based on tip flattened micro-dome shaped PI film and CNT via conventional lithography, RIE etching, and lift-off process. The sensor presents a sensing range of $3 \mathrm{MPa}$, a maximum sensitivity of $5.66 \times 10^{-3} \mathrm{KPa}^{-1}$ at $50 \mathrm{KPa}$ and a minimum sensitivity of $0.23 \times 10^{-3} \mathrm{KPa}^{-1}$ at $3 \mathrm{MPa}^{32}$. However, these PI/CNT pressure sensors cannot fulfill the above-mentioned requirements.

Herein, a facile and robust electrophoretic deposition (EPD) process is used to deposit functionalized carbon nanotubes (FCNTs) on PI fiber-based fabrics under an electric field. The fabricated PI fiber fabrics possess a large compressible region (from 'loose' PI fiber fabric to 'compact' PI fiber fabric), which is very beneficial to the sensor's linear range and sensing range. Within the PI fiber-based fabrics, plenty of micro- and nano-hierarchical pores built by the multilayer PI microfiber networks endow the matrix 
material with a large specific surface (more contacting points), high structural stability, and high flexibility (Figure 1b, Figure S1). After the EPD process, the FCNTs are chemically bonded to the PI fiber surface, forming a conductive hierarchical network (Figure 1b). Thanks to the large compressible region of PI fiber fabric, abundant yet firm contacting points, point-to-point contacting mode (Figure 1c), and high elastic modulus of both $\mathrm{PI}$ and $\mathrm{CNT}$, we demonstrate a thin $(\sim 0.33 \mathrm{~mm})$, lightweight $\left(86.5 \mathrm{mg} / \mathrm{cm}^{3}\right)$, flexible and wearable pressure sensor that is able to detect pressures from below $100 \mathrm{~Pa}$ all the way up to $45 \mathrm{MPa}$, which corresponds to the range from faint pressure (airflow, the weight of an aluminum particle) to low pressure (pulse beat, joint movement) to very high pressure (the weight of an automobile). Furthermore, thanks to the intrinsic temperature resistance of both PI and CNT, the proposed sensor also exhibit a sensitivity of 38.66 and $2.85 \mathrm{MPa}^{-1}$ in the wide linear ranges of $0-36 \mathrm{KPa}$ and $36 \mathrm{KPa}-6.88 \mathrm{MPa}$ at $200^{\circ} \mathrm{C}$ and can work at higher temperatures above $300^{\circ} \mathrm{C}$, indicating its high applicability in harsh hightemperature and high-pressure environments (Figure 1d).

\section{Results}

In order to obtain a uniform and conductive hierarchical network based on PI fiber fabric/CNT structure, four sets of pressure sensors, namely PI/CNT(Stirring), PI/FCNT(Stirring), PI/CNT(EPD), and $\mathrm{PI} / \mathrm{FCNT}(\mathrm{EPD})$, are designed and fabricated. First, a conventional mechanical stirring method was used to incorporate CNT into the PI fiber fabric (Figure 1e, PI/CNT(Stirring)). As shown in the right session of Figure $1 \mathrm{e}$, only part of the PI fiber fabric turns black while other regions remain unchanged. The underlying reason for this non-uniformity is due to the agglomeration behavior of CNT during the stirring process $^{33}$, as confirmed in Figure $1 \mathrm{f}-1 \mathrm{~h}$. Furthermore, these agglomerated CNT tend to be gathered in the gaps of the PI fibers rather than the PI surface (Figure 1f-1h, Figure S2a). To improve the uniformity, a highly acidic and thermal oxidation process was used as the initial step to modify the CNT. This process not only was beneficial to the wettability and dispersibility of the CNT, but also made the CNT functionalized with negatively charged carboxyl group ${ }^{34,35}$. An EPD process ${ }^{36}$ was then adopted to deposit FCNT on PI fiber fabric (Figure 1i). Under an electric field, these FCNTs migrated to the PI fiber fabric (anode) and were evenly wrapped to the fiber surface (Figure S3a), as evidenced by the optical and SEM images of PI/CNT(EPD) (Figure 1j-1I). It is worth noting that benefiting from the high-porous PI fiber fabric structure, these FCNTs would migrate to each fiber and be uniformly coated onto the fiber surface, even for the fibers adhered to the anode (Figure S3b-d). Figure S2b and Figure S4a represent the doping mechanism and the morphology of PI/FCNT(Stirring) and PI/CNT(EPD), respectively. Compared to $\mathrm{PI} / \mathrm{CNT}$ (Stirring), FCNT is relatively evenly distributed on the PI fiber surface for PI/FCNT(Stirring), however, the coverage degree is still far below that of PI/FCNT(EPD). The least effective doping method is the EPD process with CNT. It is observed that only a thin layer of CNTs was adhered to one surface (away from the anode) of the PI fiber fabric (Figure S4b). This is well understood because CNT cannot move directionally to the PI fibers under an applied electric field. In general, the optical and SEM morphology images show that the best strategy for obtaining a uniform and conductive network is through adopting the modified CNT with the EPD process. It is also worth noting that a 'self-assembled' method can also be adopted to develop the uniform and conductive PI/CNT network, however, the 
process may be complex and time-comsuming ${ }^{37}$. Conceivably, compared to the other three kinds of pressure sensors, the abundant contacting points within the conductive hierarchical network will be very beneficial to enhance the sensitivity, sensing range, and reliability of the PI/FCNT(EPD) pressure sensor.

The above results indicate that the modified CNT plays an important role in the proposed PI/FCNT(EPD) pressure sensor. To further probe into the intrinsic reason, we explored the interaction between PI fiber and FCNT, physical properties, and structure difference between FCNT and CNT by transmission electron microscopy (TEM), Raman spectroscopy, Fourier transform infrared (FTIR) spectroscopy, and X-ray photoelectron spectroscopy (XPS). Figure 2a-c and 2d-f are the TEM images of CNT and FCNT, respectively. The tube diameter is decreased from $\sim 16 \mathrm{~nm}$ for CNT to $\sim 8 \mathrm{~nm}$ for FCNT, which is helpful to improve the dispersion ${ }^{38-39}$. This partly accounts for the better uniformity for PI/FCNT(Stirring) compared with $\mathrm{PI} / \mathrm{CNT}$ (Stirring). Raman spectra of CNT and FCNT are shown in Figure 2g. Two characteristic peaks, located at $1321 \mathrm{~cm}^{-1}$ (D band) and $1587 \mathrm{~cm}^{-1}$ (G band), are observed for CNT. After modification, it shows a similar Raman spectrum ( $D$ band and $G$ band) but with a more distinguishable $D+$ band (1607 $\mathrm{cm}^{-1}$ ). Furthermore, FCNT possesses a narrower full-width at half maximum (FWHM) of $61.7 \mathrm{~cm}^{-1}$ compared with that of as-received CNT $\left(66.2 \mathrm{~cm}^{-1}\right)$. This is because the modification process improves the structural order and purity of the $\mathrm{CNT}^{40}$. Here, the $\mathrm{D}$ and $\mathrm{D}+$ bands represent a double-resonance Raman mode due to the amorphous carbon, disorder, defects, or ion intercalation between the graphitic walls. The $\mathrm{G}$ band is due to the tangential in-plane stretching vibrations of the carbon-carbon bonds within the graphene sheets.

FTIR spectroscopy analysis of the FCNT provides the direct evidence of the successful modification with CNT (Figure S5 and 2h), showing the characteristic band at 1536, 1573, and $1730 \mathrm{~cm}^{-1}$ which are due to $\mathrm{C}=0$ stretching vibration within the carboxyl and carbonyl functional groups ${ }^{41}$. Note that these characteristic peaks are not observed in CNT. Other notable peaks such as 1386 and $1634 \mathrm{~cm}^{-1}$ observed in both CNT and FCNT are ascribed to the $\mathrm{C}-\mathrm{O}$ and $\mathrm{O}-\mathrm{H}$ stretching vibration, respectively.

The existence of strong chemical interactions between FCNT and PI can be deduced from the Raman spectra, XPS, and FTIR analysis, as shown in Figure 2i-k. As for the Raman spectra shown in Figure 2i, two characteristic peaks of $\mathrm{PI}$ at 1377 and $1620 \mathrm{~cm}^{-1}$ are observed, corresponding to the stretch vibration of $\mathrm{C}-\mathrm{N}$ within the imide ring and the aromatic imide ring vibrations of the dianhydride portion (green line). As excepted, PI/FCNT(EPD) Raman spectra (red line) yielded a Raman spectrum containing the D-, G- and D+ bands of FCNT, which moved to a high frequency position compared to that of FCNT. In general, the Raman spectra of PI/FCNT(EPD) overlap and broaden the characteristic peaks of PI and FCNT, which is mainly attributed to the charge transfer between PI and FCNT. The average of the charge density between them causes the peak overlap and broadening ${ }^{31,41-42}$. The XPS spectra in Figure S6 show the characteristic peaks of both PI and PI/FCNT(EPD) centered at C1s (285.2 eV), N1s (400 eV), and 01s (532 $\mathrm{eV}$ ). Compared with $\mathrm{PI}$, the PI/FCNT(EPD) demonstrates an increment of $\mathrm{C} 1 \mathrm{~s}$ and 01 s peak intensity and decrement of N1s peak intensity, which is owing to the existence of FCNT. Figure 2j represents the $\mathrm{C} 1 \mathrm{~s}$ spectrum, the relative contributions of the $\mathrm{C}-\mathrm{C}, \mathrm{C}-\mathrm{N}, \mathrm{C}-\mathrm{O}, \mathrm{C}=\mathrm{O}$ are $54.11 \%, 21.26 \%, 14.38 \%, 10.24 \%$ for $\mathrm{PI}$, 
and $59.79 \%, 16.82 \%, 12.62 \%, 10.77 \%$ for PI/FCNT (EPD). There is a decreased intensity of the C-N and C$O$ for PI/FCNT(EPD) compared to that of the PI, which shows the existence of chemical interactions between FCNT and $\mathrm{PI}^{34}$. FTIR analysis of PI and PI/FCNT (EPD) was further performed, as displayed in Figure $2 \mathrm{k}$. Several notable absorption peaks located at $1370,1499,1714$, and $1776 \mathrm{~cm}^{-1}$ are clearly observed for $\mathrm{Pl}$, caused by $\mathrm{C}-\mathrm{N}, \mathrm{C}=\mathrm{C}$ stretching vibration, $\mathrm{C}=\mathrm{O}$ symmetric, and asymmetric stretching, respectively. For PI/FCNT, these absorption peaks shifted to a low wavenumber direction, and the intensity is significantly reduced due to the strong interaction between PI and FCNT. These strong chemical interactions come from the created strong hydrogen bonds between $\mathrm{C}=\mathrm{O}$ and $\mathrm{C}=\mathrm{N}$ of imide ring and carboxyl groups of FCNT ${ }^{31,41-42}$. In a word, the obtained uniform and conductive PI/FCNT (EPD) film is attributed to the directional migration of FCNT during the EPD process as well as the formed strong hydrogen bonds between $\mathrm{PI}$ and FCNT. Figure $2 \mathrm{I}$ shows the thermogravimetric analysis curves of $\mathrm{PI}$ and $\mathrm{PI} /$ FCNT (EPD). At low temperature, the weight loss for PI/FCNT(EPD) is higher than that of PI. This is due to the high conductivity of CNT, which acts as a heat source to induce the decomposition of $\mathrm{PI}^{31}$. It is worth noting that although the thermal stability of the PI/FCNT(EPD) film is decreased, its $\mathrm{T}_{5 \%}$ (the temperature at $5 \%$ weight loss, $T_{5 \%}$ ) still exceeds $350^{\circ} \mathrm{C}$, revealing its excellent thermal stability.

Next, we consider the sensing performance of the proposed four sets of pressure sensors. To better understand the sensing mechanism for the PI fiber/CNT architectures and compare their sensing performance, Creo/Engineering software is adopted to build the PI fiber/CNT model and finite element modeling (FEM) to analyze the dynamic working process of the pressure sensors under external pressure. A detailed explanation of the FEM simulation can be found in the Supporting Information (Figure S7-S9). Figure S10a-c shows the established models for PI/CNT(stirring), PI/FCNT(stirring), and PI/FCNT(EPD), respectively. Note that the $\mathrm{PI} / \mathrm{CNT}$ (EPD) pressure sensor is not taken into consideration, because there is only a thin layer of CNT adhered to one surface of the PI fiber fabric (Figure S4b), and the conductive network is not formed. According to the SEM results shown in Figure 1, Figures S2-S3, the established conductive contact mode for PI/CNT(stirring), PI/FCNT(stirring), and PI/FCNT(EPD) is CNT cluster to CNT cluster, CNT cluster to PI/FCNT fibers, PI/FCNT fibers to PI/FCNT fibers (Figure S10a-c), respectively. Dynamic microscopic deformation processes for these models are displayed in Figures $3 \mathrm{a}-\mathrm{c}$ and Figures S11-S13. Figure 3d shows the contacting area as a function of time for the four sets of pressure sensors. As the time increases from 0 to $1 \mathrm{~s}$, the increment speed of the number contacting for PI/FCNT(EPD) is far above that of the other three types of pressure sensors, indicating that the PI/FCNT(EPD) pressure sensor possesses the highest sensitivity. Besides, the contacting area gradually increases without saturation as the external pressure exceeding $2 \mathrm{MPa}$, revealing a broad working range for the proposed PI/FCNT(EPD) pressure sensor. The theoretical results presented in Figure $3 \mathrm{~d}$ and $3 \mathrm{e}$ agree well with the experimental results as detailed below.

To experimentally evaluate the performance of the proposed pressure sensors, we set up an intelligent data-acquisition device containing a universal electric signal analyzer Keithley 2400 and a computercontrolled dynamic positioning system. The effects of the CNT morphology and doping technique on the sensitivity of the proposed pressure sensors were studied by measuring the output current change as a 
function of applied external pressure, as shown in Figure 4a-b. The size of the pressure sensors is $5 \mathrm{~mm}$ (length) $\times 5 \mathrm{~mm}$ (width) $\times 0.33 \mathrm{~mm}$ (thickness of the encapsulated sensor). Note that the thickness of the PI fiber fabric is $210 \mu \mathrm{m}$, and all the characterization and application shown in the paper are based on this type of PI fiber fabric. As expected, the PI/FCNT(EPD) pressure sensor exhibits higher current change under the same external pressure (namely, higher sensitivity) and broader sensing range than that of other sets of pressure sensors, showing an excellent agreement with the theoretical prediction (Figure 3 ). $\mathrm{PI} / \mathrm{FCNT}$ (EPD) pressure sensor exhibits wide linearity and an exceptionally broad sensing range up to 45 MPa. In addition, the fabricated PI/FCNT(EPD) pressure sensors exhibit reliable pressure responses with good uniformity, as shown in Figure S14 (3 PI/FCNT (EPD) pressure sensor). The sensing behavior of the PI/FCNT(EPD) pressure sensors can be divided into two stages (Figure 3b). To explain this, an illustration of the equivalent circuit to show the resistance changes under different pressures is shown in Figure 4c-d. In the low-pressure region (0-3.38 MPa), the PI fiber fabric experienced the densification process, namely, from 'loose' PI fiber fabric to 'compact' PI fiber fabric. In this region, the point-to-point contact working mechanism played the leading role in the resistance change, and the pressure sensor possessed a relatively high sensitivity because of a large amount of fibers contact, leading to the result that the resistance changed abruptly: from infinite $(+\infty)$ to $\mathrm{Rc}$. In the high-pressure region (3.38 $\mathrm{MPa}-45 \mathrm{MPa})$, the $\mathrm{PI} / \mathrm{FCNT}$ (EPD) was almost densified. In this region, the resistance change was mainly dependent on the deformation of the PI/FCNT fibers, namely, the 'surface-to-surface' contact played the primary role in the sensitivity.

We attribute this wide linearity and ultra-broad response range to the large yet appropriate compressible region of PI fiber fabric, abundant yet firm contacting sites (high-density FCNT is evenly wrapped and chemically bonded to the PI fibers via EPD process), point-to-point contacting mode within the hierarchical conductive network, and high elastic modulus of both PI and CNT. The result of wide linearity and broad sensing range is, first of all, determined by the compressible region of the PI film. To demonstrate this, two other types of PI fiber fabrics with thicknesses of 50 and $100 \mu \mathrm{m}$ are designed and fabricated (Figure S15). As shown from the compressive stress-strain curve (Figure S16), the compressible region enlarges with the increase of the PI fiber fabric thickness. We fabricated pressure sensors based on these three types of PI fiber fabrics. The P-I results are shown in Figure S17, demonstrating that the linearity of the pressure sensor is proportional to the compressible region of the PI fiber fabric. In other words, a large compressible region for the PI fiber fabric $(210 \mu \mathrm{m})$ contributes to the wide linearity. It needs to mention that for the PI/FCNT (EPD) (PI fiber fabric: $210 \mu \mathrm{m}$ ) pressure sensor, there is a very short high sensitivity region (0-6 KPa, corresponding to $0-0.15 \mathrm{~N})$, which may be due to the 'rapid densification' of the PI fiber fabric during the initial compressive process (Figure 4e-f). The 'rapid densification' region is originated from the relatively low effective modulus of the porous $\mathrm{PI}$ fiber fabric. However, for the PI fiber fabrics with the thickness of $50 \mu \mathrm{m}$ and $100 \mu \mathrm{m}$, the 'rapid densification' region is not observed. This is due to the following two reasons: (1) the 'rapid densification' region of the thin PI fiber fabric is shorter than that of the thicker PI fiber fabric (Figure S16); (2) this short 'rapid densification' region is further decreased due to the initial imposed pressure during the packaging process. In order to verify the above analysis, we further fabricated the pressure sensor with 
thicker PI fiber fabric by stacking two PI fiber fabric $(210 \mu \mathrm{m})$. As excepted, the 'rapid densification' working region of the pressure sensor becomes larger (Figure S18). In other words, the whole linearity is decreased. As a result, PI/FCNT (EPD) pressure sensor based on the PI fiber fabric with the thickness of $210 \mu \mathrm{m}$ is the optimal selection, which combines the merits of thin, lightweight, flexible, wide linearity, and ultra-broad sensing range. Besides, as mentioned above, PI film with a wide compressible region is an essential prerequisite for realizing high linearity, however, this is not a sufficient condition. Another important factor is the point-to-point contacting mode, which offer large and consistent resistance change during the 'loose' PI fiber fabric to 'compact' PI fiber fabric region.

Our proposed $\mathrm{PI} / \mathrm{FCNT}(\mathrm{EPD})$ pressure sensor not only can work at high pressure but also can operate at high temperatures. Figure S19 are the infrared (IR) thermal imaging images of the PI/FCNT(EPD) pressure sensor placed on a heat source, respectively. It can be seen that even if the heating temperature exceeds $300^{\circ} \mathrm{C}$, the morphology and the temperature distribution of the PI/FCNT(EPD) pressure sensor almost remains the same during a heating period of $5 \mathrm{~min}$. The sensing performance of the $\mathrm{PI} / \mathrm{FCNT}$ (EPD) pressure sensor at $200^{\circ} \mathrm{C}$ is also characterized (see Methods), as depicts in Figure S20. Note that before the testing, the PI/FCNT (EPD) film was baked at $200^{\circ} \mathrm{C}$ for $48 \mathrm{~h}$ for the aging process. The proposed sensor device exhibits stable P-I curves (Figures S21), and still possesses a sensitivity of 38.66 and $2.85 \mathrm{MPa}^{-1}$ in the wide linear ranges of $0-36 \mathrm{KPa}$ and $36 \mathrm{KPa}-6.88 \mathrm{MPa}$. Overall, these hightemperature experimental results demonstrated the flame retardancy and good heat stability of the proposed $\mathrm{PI} / \mathrm{FCNT}$ pressure sensors, enabling their potential to be used in harsh high-temperature conditions.

Besides the sensitivity and sensing range, another electric characteristic of the PI/FCNT(EPD) pressure sensors was also tested. Figure $4 \mathrm{~g}$ is the current-voltage (I-V) curves of the PI/FCNT(EPD) under various applied pressures. The I-V curves exhibit high linearity, revealing their excellent ohmic performance independent of the applied voltage. Figure $4 \mathrm{~h}$ represents the current response of the PI/FCNT(EPD) pressure sensor over five on/off cycles under various external pressures (1.1 MPa, 1.3 MPa, 1.6 MPa, 2 $\mathrm{MPa}$, and $7 \mathrm{MPa}$ ). The current experienced fast-changing under the repeated pressure loading and relaxation cycles. Besides, the current remained unchanged during the pressure loading process, indicating the robust repeatability and excellent reliability of the PI/FCNT(EPD) pressure sensors independent of the applied pressures. We further explored the sensors' durability by repeated loading and unloading pressure of $1.1 \mathrm{MPa}$ for more than 4000 cycles, as shown in Figure 4i. It is found that the sensor maintained its function with minimal output signal degradation. This excellent endurance behavior can be attributed to the strong chemical interaction between PI and FCNT as well as the excellent compressibility of both PI fiber fabric and CNT. To demonstrate this, the compressive mechanical properties of the PI/FCNT(EPD) nanofibrous network under cyclic compressive stress-strain was characterized. Figure $S 22$ shows cyclic compressive stress-strain curves (1000 cycles) for the PI/FCNT(EPD) nanofibrous network at room temperature. Note that the tested PI/FCNT(EPD) nanofibrous network is encapsulated by PI film, and the encapsulation process is the same as that of the $\mathrm{PI} / \mathrm{FCNT}$ (EPD) pressure sensor (See the methods), which can better reflect the robustness of sensing 
performance of the PI/FCNT(EPD) pressure sensor. As shown in Figure S22, the PI/FCNT(EPD) nanofibrous network maintains over $91 \%$ of its initial maximum stress after 1000 cycles, demonstrating its superior compressive properties, ensuring a reliable and robust performance for long-term applications. In addition, the fabricated sensor exhibits a fast rise time of $100 \mathrm{~ms}$ and a relaxation time of $80 \mathrm{~ms}$ (Figure 4j), which also indicates that the force unloading process is faster than that of the loading process. The response time is mainly determined by the elastic recovery of the PI fiber fabric. Hysteresis of the PI/FCNT(EPD) pressure sensor (linear region) is characterized as shown in Figure S23, and the sensor exhibits a hysteresis of $5.5 \%$. The residual resistance is about $2.6 \%$. The hysteresis and residual resistance may be due to the viscoelastic behavior of the PI. However, the hysteresis is relatively small, which may be attributed to the following two reasons: (1) large elastic modulus of the PI materials (PI fiber fabric and PI encapsulation layer); (2) the area of the electrode is independent of the sensing area (eliminating the effect of hysteretic behavior of the adhesive which is used to attach the sensing area and the electrode in the conventional vertical-structured pressure sensor). Finally, the electric characteristics including I-V linearity and reliability of the sensor under $100^{\circ} \mathrm{C}$ were also tested. As shown in Figure $4 \mathrm{k}-\mathrm{I}$, the results demonstrate high linearity and excellent reliability of the PI/FCNT(EPD) pressure sensor, further endowing it with high applicability in high-temperature environments.

These sensing characterizations and analyses demonstrate that the proposed PI/FCNT(EPD) pressure sensor exhibits superior sensing performance including wide linearity, exceptionally broad sensing range, and high-temperature-resistant properties. The wide linearity and relatively high sensitivity reveal that the resulting sensor can act as an ideal candidate to detect the faint pressure (<100 $\mathrm{Pa}$ ), low-pressure (in the range of $\mathrm{KPa}$, such as human motions), and high pressure (in the range of MPa). To testify the sensing capability, we conducted the following experiments and further explored their applications in real life. First of all, as shown in Figure 5a, a small meter screw $(0.161 \mathrm{~g}, \sim 63.1 \mathrm{~Pa})$ is put on the sensor. The corresponding current change is displayed in Figure $5 \mathrm{~b}$ (red line), which shows that such a tiny pressure change can be precisely detected. Moreover, the limit of detection of our sensor device was also measured to be $\sim 8.2 \mathrm{~Pa}$, as depicted in Figure 5b (blue line, an aluminum particle, $0.021 \mathrm{~g}$ ). Furthermore, a sharp current response for airflow again shows the capability of detecting the faint pressure (Movie S1, Movie S2, Supporting information). Secondly, the sensor's ability as a skin-mountable human motion detector is explored. For the test, the fabricated PI/FCNT(EPD) pressure sensor was attached to various parts of the human body, as shown in Figure 5c-f. As a result, the fabricated PI/FCNT(EPD) pressure sensor is highly responsive to the repetitive dynamic flexion and straightening motions of the finger joint, wrist, elbow, and ankle. Besides, the cycling tests show that the response and relaxation behaviors are reproducible. The flexible PI/FCNT(EPD) pressure sensor can also be used to make a pulse sensor for the detection of the radial artery pulse (Figure S24). The above results clearly suggest that the human motions can be identified with the PI/FCNT(EPD) pressure sensor, enabling its potential to monitor human physiological information in real-time.

To further improve the practicability of the PI/FCNT(EPD) pressure sensors, a real-time, wireless pressure monitoring system is developed, including data acquisition, wireless data transmission, and display via 
APP interface on a mobile phone, as displayed in Figure $5 \mathrm{~g}$. Here, the function of the processing unit shown in Figure $5 \mathrm{~g}$ involves the data acquisition collected from the pressure sensor, analog-digital conversion, and wireless data transmission. With the real-time pressure monitoring system, two highpressure experiments were carried out: (1) the PI/FCNT(EPD) pressure sensors was first compressed to a reference high-pressure of $15.8 \mathrm{MPa}$, followed by adding a metal with weighing about $\sim 3.8 \mathrm{Kg}$, which is equivalent to a pressure increment of $1.5 \mathrm{MPa}$. The real-time pressure monitoring during the metal loading and unloading process is displayed by the APP interface, as shown in the right session of Figure $5 \mathrm{~g}$. The results show that the PI/FCNT(EPD) pressure sensor can detect relatively small changes in pressure under extremely high pressures, even though the sensor is in a non-linear state (as shown in Figure $4 b$ ). This significantly extends its available pressure sensing range, demonstrating its high potential for precise real-time detection of extremely high pressure. (2) Another experiment is the pressure detection of a vehicle during the running process (Figure 5h). The PI/FCNT(EPD) pressure sensor is repeatedly rolled by the front wheel of a car. The recorded real-time pressure data is collected from APP and displayed in Figure 5i. When the car is driven over the sensor, the pressure increases instantaneously and returns to its original state without any noticeable time lag. Besides, the sensor remained its function after the repeated rolling process. This greatly enables its application in the industry, such as safety monitoring of road, rail, bridge, and tunnel.

An ideal intelligent robot should not only require the basic capability of sensing force and pressure, but also can collect some characteristic data in some harsh high-temperature conditions (for example, firefighting). As a proof of concept, we designed the following experiments. We wrapped the $\mathrm{PI} / \mathrm{FCNT}$ (EPD) pressure sensor in the fingertips of a mechanical hand while the mechanical hand was mounted on a computer-controlled stepping motor. The mechanic hand with the conformally wrapped sensor moved forward to a high-temperature object $\left(300^{\circ} \mathrm{C}\right)$. When the sensor device touched the surface of the hot object, the output current increased. Here, a threshold current was set for the stepping motor. The stepping motor stopped and moved backward when the output current of the sensor device reached the threshold current. Figure $5 \mathrm{j}$ and $5 \mathrm{k}$ show the IR images as the mechanic hand moved close to and touched the hot object, respectively. The temperature of the fingertip increased from the room temperature to $81.5^{\circ} \mathrm{Cand} 249^{\circ} \mathrm{C}$, respectively. Figure $5 \mathrm{l}$ shows the current responding curve of the $\mathrm{PI} / \mathrm{CNT}$ (EPD) pressure sensor during the 'close, touch, feel, and response' process. It is observed that the current rises steeply to the threshold value and recovers quickly, demonstrating its great potential to serve as a wearable and high sensitivity pressure sensor that is able to withstand harsh environment where high temperature is present.

Ploy (ether-ether-ketone) (PEEK) is a special engingeering plastics and usually adopted as the protecting sheath of the motor rotor benefiting from its superior properties such as good resistant properties to chemical corrosion, et al. During the winding process (Figure 6), the PEEKs will stick together under the high power laser heating process $\left(380^{\circ} \mathrm{C}\right)$, forming a huge tension imposed on the motor rotor. During the actual operation process, it is vital to detect the pre-stresses within the composite sheath of the motor rotor for the high-speed permanent magnet motor rotor to ensure the safety of the whole system. For the 
pre-stresses monitoring sensor, several points should be addressed: (1) the sensor should be flexible, thin and can be integrated into the motor rotor; (2) the sensor should be high-temperature resistant because there is a (laser) heating process; (3) the sensor can work at high-pressure environment due to the large tension. Herein, we show that the fabricated PI/FCNT(EPD) pressure sensor can act as a promising candidate for the real-time detection of the pre-stresses. Figure $6 a-b$ shows the final PI/FCNT (EPD) pressure sensor with PEEK encapsulation, the whole structure is PEEK-Cu electrode-PI/FCNT(EPD) film-Cu electrode-PEEK. Note that before the encapsulation process, the PI/FCNT (EPD) film was aged at $400{ }^{\circ} \mathrm{C}$ for $48 \mathrm{~h}$. Figure $6 \mathrm{c}-\mathrm{d}$ is the experimental setup for the PEEK winding process. The applied pressure on the PI/FCNT (EPD) pressure sensor come from two parts, the roller and the PEEK. Figure 6e shows the corresponding P-I properties of the PI/FCNT(EPD) pressure sensor. It is observed that the repeatability is robust up to $22.5 \mathrm{MPa}$ during the cycling test, demonstrating its potential use in both harsh high temperature and high pressure environment.

\section{Discussion}

The performances of the recently reported high-performance pressure sensors are summarized in Table S1. Superior comprehensive properties including thin, lightweight, broad working range, high temperature resistance, the capability of the detection of full-range pressure (from $<100 \mathrm{~Pa}$ to $\sim \mathrm{MPa}$ ) are found in the proposed PI/FCNT(EPD) pressure sensor. In addition, this large-scale fabrication process of PI fiber fabrics, the proposed facile and robust EPD process, and the developed data transmission system are very beneficial to enable the technology transfer from the laboratory to the industry. The future scope for this study is to further increase the sensitivity. For example, appropriately reducing the Young's modulus of $\mathrm{PI}$ fiber fabrics (more point contacts variation, $\Delta \mathrm{I} / \mathrm{IO} \uparrow$ ) via optimizing the solution formula and electrospinning process would address this issue, as well as ensuring broad linearity. Moreover, although the current application of the fabricated PI/FCNT(EPD) sensors focuses on the daily activities and harsh high temperature and high-pressure condition, these thin, lightweight, flexible textile pressure sensors and the corresponding wireless pressure sensing system are ready to be used in practical biomedical applications, because they are resistant to most chemical attacks.

\section{Conclusion}

In conclusion, we have developed a simple, robust, and effective method via modification process and EPD method for fabricating high-performance wearable pressure sensors. High-density FCNT is evenly wrapped and chemically bonded to the PI fibers via the EPD process. Benefiting from the large yet appropriate compressible region of PI fiber fabric, abundant yet firm contacting points, point-to-point contacting mode within the hierarchical conductive network and high elastic modulus of both PI and CNT, the proposed PI/FCNT pressure sensor possesses the merits of thin, lightweight, wide linearity, exceptionally broad sensing range, and long-term stability. Furthermore, thanks to the high-temperatureresistant properties of both $\mathrm{PI}$ and $\mathrm{CNT}$, the PI/FCNT pressure sensor device still exhibits a sensitivity of 38.66 and $2.85 \mathrm{MPa}^{-1}$ in the wide linear ranges of $0-36 \mathrm{KPa}$ and $36 \mathrm{KPa}-6.88 \mathrm{MPa}$. Besides, we have 
developed a real-time, wireless pressure monitoring system and demonstrated that the pressure sensor could serve as an ideal platform to monitor the human physiological information, precisely detect extremely high pressure, and can be integrated into an intelligent mechanical hand to detect the contact force under the harsh high-temperature environment.

\section{Materials And Methods}

\section{Fabrication of PI/FCNT(EPD) Piezoresistive Pressure Sensor.}

FCNT was obtained by the modification process of CNT. CNT was purchased from XFNANO Co., Ltd. Next, $1.044 \mathrm{~g}$ CNTs were dispersed in $40 \mathrm{ml}$ of the mixture of concentrated sulphuric acid $(30 \mathrm{ml})$ and concentrated nitric acid $(10 \mathrm{ml})$ in a flask. The mixture was then stirred using a magnetic stirrer at $150^{\circ} \mathrm{C}$ for $1 \mathrm{~h}$. The resulting dispersion was then diluted in water, and filtered repeatedly until the solution $\mathrm{PH}=7$, followed by the drying process in vacuum at $40^{\circ} \mathrm{C}$ overnight. The detailed fabrication process of PI fiber fabric and PI films can be found in our previous work ${ }^{43}$. Briefly, poly(amic acid) (PAA) (10 wt\%) was synthesized by an equimolar ratio of 3, 3', 4, 4'-Biphenyltetracarboxylic dianhydride(BPDA) and 4, 4'Oxydianiline(ODA) in N, $\mathrm{N}^{\prime}$-dimethylacetamide (DMAc) at room temperature for $24 \mathrm{~h}$. The PAA solutions were diluted to $6 \mathrm{wt} \%$ by DMAc. The average electrical field was $100 \mathrm{kV} / \mathrm{m}$ by imposing $20 \mathrm{kV}$ electrical potential into a $20 \mathrm{~cm}$ gap between spinneret and collector. After removing the residual solvent in a vacuum oven at $75^{\circ} \mathrm{C}$ for $8 \mathrm{~h}$, the sample was imidized by the following processes: (1) heating up to 100 ${ }^{\circ} \mathrm{C}\left(5^{\circ} \mathrm{C} / \mathrm{min}, \mathrm{N}_{2}\right)$ and annealing for $1 \mathrm{~h}$; (2) heating up to $200{ }^{\circ} \mathrm{C}\left(2^{\circ} \mathrm{C} / \mathrm{min}, \mathrm{N}_{2}\right)$ and annealing for $0.5 \mathrm{~h}$; (3) heating up to $380^{\circ} \mathrm{C}\left(2{ }^{\circ} \mathrm{C} / \mathrm{min}, \mathrm{N}_{2}\right)$ and annealing for $0.3 \mathrm{~h}$; (4) cooling down to the room temperature. Functional carbon nanotube dispersions were used to electrophoretically deposit nanotubes onto the PI fiber fabric. $0.1046 \mathrm{~g}$ FCNT was dispersed in $100 \mathrm{ml}$ anhydrous alcohol and then stirred using magnetic for $7 \mathrm{~h}$. The PI fiber fabric was placed in contact with a stainless steel cathode as the anode. The same stainless steel was placed a fixed distance from the anode. EPD process was carried out under direct current with the voltage of $30 \mathrm{~V}$ for $1 \mathrm{~h}$. The PI/FCNT fiber fabric was then dried in an oven for 10 min at $60^{\circ} \mathrm{C}$. After the EPD and process, the PI/FCNT fiber fabric was cut into the designed concave shape, as shown in Figure 1a and 1c. Cu wire electrodes were adhered to the bulgy area of the sensor through the conductive silver paste.

\section{Fabrication of PI/CNT (Stirring), PI/FCNT(Stirring), and PI/CNT(EPD) Piezoresistive Pressure Sensor.}

PI/CNT (Stirring) pressure sensor: $0.1046 \mathrm{~g}$ CNT was dispersed in $100 \mathrm{ml}$ anhydrous alcohol. Then the PI fiber fabric was immersed in the CNT dispersion and stirred using a magnetic stirrer for $7 \mathrm{~h}$. The subsequent baking and tailoring process was the same as PI/FCNT (EPD). PI/FCNT (Stirring): Compared with the PI/CNT (Stirring) pressure sensor, besides the difference of the functional layer (FCNT instead of $\mathrm{CNT}$ ), the other process was the same. PI/CNT (EPD): Compared with the PI/FCNT (EPD) pressure sensor, besides the difference of the functional layer (CNT instead of FCNT), the other process was the same. 


\section{Encapsulation, characterization, test, and simulation.}

First, the PI/FCNT (EPD) film was placed between two pieces of PI film, forming a sandwich structure. Then this sandwich structure was further encapsulated by the PI adhesive tape. The thickness of the encapsulated PI/FCNT (EPD) pressure sensor was $\sim 0.33 \mathrm{~mm}$ (Figure S25). The 2D and 3D morphologies of the PI fiber fabric and PI/FCNT(EPD) were characterized using SEM (AEISS SUPRA55) and KEYENCE VK-X1000. The morphologies of CNT and FCNT were further characterized using TEM (JEM-3200FS). Raman analysis was carried out on a Witec CRM200 backscattering Raman system. The compression test of the PI fiber fabrics and PI/FCNT(EPD) were carried out on a universal testing machine (SHIMADZU AG-X Plus $1 \mathrm{kN}$ ). For XPS measurement, ESCALAB 250Xi was used. For FTIR measurement, Frontier was used. TGA analysis was conducted on a thermogravimetric analyzer (Mettler Toledo, Switzerland) from room temperature to $800^{\circ} \mathrm{C}$. A semiconductor parameter analyzer Keithley 2400 was employed to measure the current-time $(I-t)$ characteristics of the pressure sensors. The cycling performance of the pressure sensor was determined using a pressure meter (Dongguan Zhiqu Precision Instrument Co., Ltd). Oscilloscope (LUCK-3, digital storage oscilloscope, Chengdu Rongte Instrument Co., Ltd.) was used to measure the response and recovery time. In order to theoretically study the sensing performance of the pressure sensors, Creo and Ansys software were employed in the simulation. PI/CNT models is designed and built via Creo software, then the established models were imported into Ansys software for further analysis. The PI fiber was modeled as a homogeneous isotropic elastic material with Young's modulus $\mathrm{E}=2.5 \mathrm{GPa}$, and Poisson's ratio $v=0.34$. For high temperature $\mathrm{P}-\mathrm{I}$ and other electric characterization, the $\mathrm{PI} / \mathrm{FCNT}$ (EPD) film was baked at $200^{\circ} \mathrm{C}$ for $48 \mathrm{~h}$ for the aging process.

\section{References}

1. Kim, J. et al. Soft wearable pressure sensors for beat-to-beat blood pressure monitoring. Adv. Healthc. Mater. 8, e1900109 (2019).

2. Schwartz, G. et al. Flexible polymer transistors with high pressure sensitivity for application in electronic skin and health monitoring. Nat. Commun. 4, 1859 (2013).

3. Boutry, C. M. et al. A sensitive and biodegradable pressure sensor array for cardiovascular monitoring. Adv. Mater. 27, 6954 (2015).

4. Tang, X. et al. Multilevel microstructured flexible pressure sensors with ultrahigh sensitivity and ultrawide pressure range for versatile electronic skins. Small 15, e1804559 (2019).

5. Rus, D. \& Tolley, M. T. Design, fabrication and control of soft robots. Nature 521, 467 (2015).

6. Liu, M. et al. Large-area all-textile pressure sensors for monitoring human motion and physiological signals. Adv. Mater. 29, 1703700 (2017).

7. Wang, X. et al. Recent Progress in Electronic Skin. Adv. Sci. 2, 1500169 (2015). 
8. Wang, X., Gu, Y., Xiong, Z., Cui, Z. \& Zhang, T. Silk-molded flexible, ultrasensitive, and highly stable electronic skin for monitoring human physiological signals. Adv. Mater. 26, 1336-1342 (2014).

9. Zhu, B. et al. Hierarchically structured vertical gold nanowire array-based wearable pressure sensors for wireless health monitoring. ACS Appl. Mater. Interfaces 11, 29014-29021 (2019).

10. Meng, K. et al. A wireless textile-based sensor system for self-powered personalized health care. Matter 2, 896 (2020).

11. Peng, X. et al. A breathable, biodegradable, antibacterial, and self-powered electronic skin based on all-nanofiber triboelectric nanogenerators. Sci. Adv. 6, eaba9624 (2020).

12. Lin, L. et al. Triboelectric active sensor array for self-powered static and dynamic pressure detection and tactile imaging. ACS Nano 7, 8266-8274 (2013)

13. Chen, J. et al. Soft robots with self-powered configurational sensing. Nano Energy 77, 105171 (2020)

14. Bai, N. et al. Graded intrafillable architecture-based iontronic pressure sensor with ultra-broad-range high sensitivity. Nat. Commun. 11, 209 (2020).

15. Luo, Y. et al. Flexible capacitive pressure sensor enhanced by tilted micropillar arrays. ACS Appl. Mater. Interfaces 11, 17796-17803 (2019).

16. Huang, Y., Fan, X., Chen. S. C. \& Zhao, N. Emerging technologies of flexible pressure sensors: materials, modeling, devices, and manufacturing. Adv. Func. Mater. 29, 1808509 (2019).

17. Yang, Y. et al. Flexible piezoelectric pressure sensor based on polydopamine-modified BaTiO3/PVDF composite film for human motion monitoring. Sensors and Actuators A: Physical 301, 111789 (2020).

18. Ma, Y. et al. A highly flexible and sensitive piezoresistive sensor based on MXene with greatly changed interlayer distances. Nat. Commun. 8, 1207 (2017).

19. Luo, C. et al. A new approach for ultrahigh-performance piezoresistive sensor based on wrinkled PPy film with electrospun PVA nanowires as spacer. Nano Energy 41, 527-534 (2017).

20. Gao, L. et al. All paper-based flexible and wearable piezoresistive pressure sensor. ACS Appl. Mater. Interfaces 11, 25034-25042 (2019).

21. Zhu, B. et al. Microstructured grapheme arrays for highly sensitive flexible tactile sensors. Small 10, 3625-3631 (2014).

22. Peng, S. et al. Rational design of ultrasensitive pressure sensors by tailoring micorscopic features. Adv. Mater. Interfaces 5, 1800403 (2018).

23. Wang, Z. et al. Highly sensitivity, wearable, piezoresistive pressure sensors based on irregrlar microhump structures and its applications in body motion sensing. Small 12, 3827-3836 (2016).

24. Jian, M. et al. Flexible and highly sensitive pressure sensors based on bionic hierarchical structures. Adv. Func. Mater. 27, 1606066 (2017).

25. He, K. et al. High-performance zero-standby-power-consumption-under-bending pressure sensors for artificial reflex arc. Nano Energy 73, 104743 (2020). 
26. Chen, M. et al. Touchpoint-tailored ultrasensitive piezoresistive pressure sensors with a broad dynamic response range and low detection limit. ACS Appl. Mater. Interfaces 11, 2551-2558 (2019).

27. Yi, C. et al. Highly sensitive and wide linear-response pressure sensors featuring zero standby power consumption under bending conditions. ACS Appl. Mater. Interfaces 12, 19563-19571 (2020).

28. Li, W. et al. Flexible and high performance piezoresistive pressure sensors based on hierarchical flower-shaped $\mathrm{SnSe}_{2}$ nanoplates. ACS Appl. Ener. Mater. 2, 2803-2809 (2019).

29. Liaw, D. et al. Advanced polyimide materials: syntheses, physical properties and applications. Prog. Polym. 37, 907-974 (2012).

30. Gouzman, I. et al. Advances in polyimide-based materials for space applications. Adv. Mater. 31, e1807738 (2019).

31. Chen, X. et al. Highly compressible and robust polyimide/carbon nanotube composite aerogel for high-performance wearable pressure sensor. ACS Appl. Mater. Interfaces 11, 42594-42606 (2020).

32. Jeong, Y. et al. Ultra-wide range pressure sensor based on a microstructured conductive nanocomposite for wearable workout monitoring. Adv. Healthc. Mater. 10, 2001461 (2021).

33. Vaisman, L. et al. The role of surfactants in dispersion of carbon nanotubes. Adv. Colloid. Interface Sci. 128, 37-46 (2006).

34. Hilding, J. et al. Dispersion of carbon nanotubes in liquids. J. Dispersion Sci. Technol. 24, 1-41 (2007).

35. Rodríguez, U. O. et al. Electrical self-sensing of strain and damage of thermoplastic hierarchical composites subjected to monotonic and cyclic tensile loading. J. Intell. Mater. Syst. Struct. 30, 15271537 (2019).

36. An, Q., Rider, A. N. \& Thostenson, E. T. Hierarchical composite structures prepared by electrophoretic deposition of carbon nanotubes onto glass fibers. ACS Appl. Mater. Interfaces, 5, 2022-2032 (2013).

37. Ehlert, G. J. \& Sodano, H. A. Fiber strain sensors from carbon nanotubes self-assembled on aramid fibers. J. Intell. Mater. Syst. Struct. 25, 2117-2121 (2014).

38. Rosca, I. D. et al. Oxidation of multiwalled carbon nanotubes by nitric acid. Carbon, 43, 3124-3131 (2005).

39. Avilés, F. et al. TEM examination of MWCNTs oxidized by mild experimental conditions. Fullerenes, Nanotubes Carbon Nanostruct. 20, 49-55 (2012).

40. Osswald, S. et al. Monitoring oxidation of multiwalled carbon nanotubes by Raman spectroscopy. J. Raman Spectrosc. 38, 728-736 (2007).

41. Qin Y, Peng Q, Ding Y, et al. Lightweight, superelastic, and mechanically flexible graphene/polyimide nanocomposite foam for strain sensor application. ACS nano. 9, 8933-8941 (2015).

42. Ramakrishnan, S. et al. Synthesis and characterization of graphene oxide-polyimide nanofiber composites. RSC Adv. 19, 9743-9749 (2014).

43. Ma, P. et al. Investigate of Mo films deposited on high temperature polyimide substrate by magnetron sputtering for flexible CIGS thin film solar cells applications. AIP adv. 9, 045024 (2019). 


\section{Declarations}

\section{Acknowledgments}

Funding: This work was partially supported by the Shenzhen Basic Research Grant:

JCYJ20200109114801744, JCYJ20180507182431967, JCYJ20180507182445460, the National Nature Science Foundation of China $(11804354,61774164,51903249)$. This work was supported in part by the Singapore Ministry of Education Academic Research Fund Tier 2 (MOE2019-T2-2-127), the Singapore Ministry of Education Academic Research Fund Tier 1 (MOE2019-T1-001-103 and MOE2019-T1-001-111) and the Singapore National Research Foundation Competitive Research Program (NRF-CRP18-2017-02). This work was also supported in part by Nanyang Technological University.

Author contributions: M. J., C. Y. and Y. H. carried out the modification and EPD experiment, performed P-I test, XPS, Raman, SEM, TEM experiments and analyzed the data. X.L. and Y. H performed the simulation. M. J., G. X., K. H., N. L., Z. W., W. L., P. T., Y. Z., J. Y. and Y. Z. carried out the experiment of the application of the pressure sensor. Y. H. carried out the compressive stress-strain experiment. M.C. and L.W. wrote the manuscript, and all authors discussed the results and commented on the manuscript.

Competing interests: The authors declare that they have no competing interests.

Data and materials availability: All data needed to evaluate the conclusions in the paper are present in the paper and/or the Supplementary Materials. Additional data related to this paper may be requested from the authors.

\section{Figures}



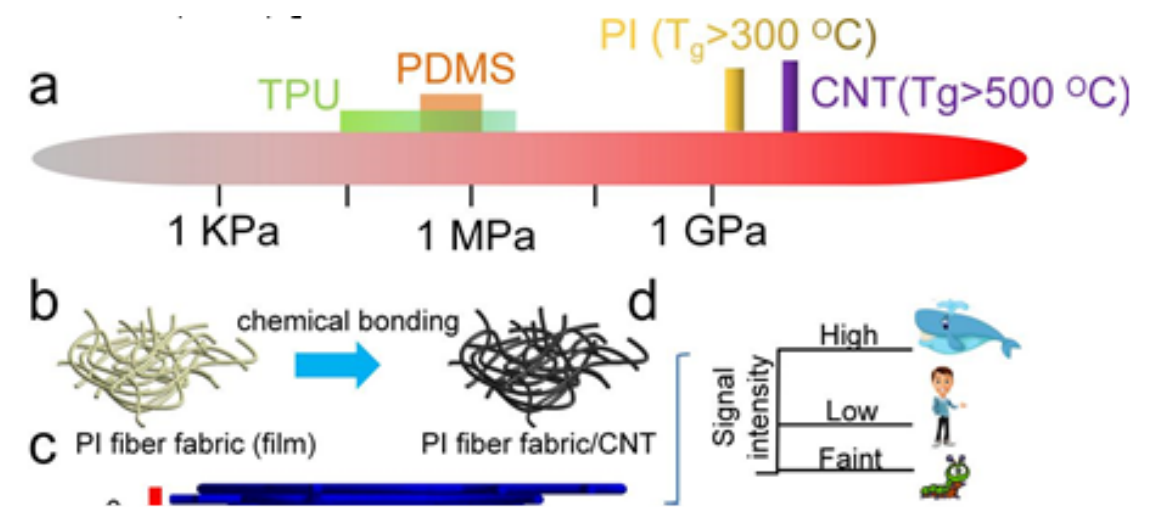

\section{Figure 1}

Materials selection, structure optimization, sensing mechanism and the fabricated PI/CNT(Stirring), PI/FCNT(EPD) pressure sensors. A, Materials selection: schematic illustration of Young's modulus of frequently used flexible matrix materials and conductive materials for the pressure sensors. $B$, Structure optimization: PI fiber-based fabrics and CNT is adopted as flexible matrix materials and conductive materials, respectively. C, Sensing mechanism: contacting points variation of the PI fiber fabrics/CNT 
under external pressure from 0-500 KPa. D, Schematic illustration of the response of the proposed PI fiber fabrics/CNT pressure sensor to faint, low, and high pressure, and such proposed pressure sensor possesses the merit of high-temperature resistance. E, Schematic illustration of the fabrication process of $\mathrm{PI} / \mathrm{CNT}$ (Stirring) fabric, optical images of the PI/CNT(Stirring) fabric, and the final encapsulated $\mathrm{PI} / \mathrm{CNT}$ (Stirring) pressure sensor. F-H, Top-view SEM images of the PI/CNT(Stirring) fabric. (i) Schematic illustration of the fabrication process of PI/FCNT(EPD) fabric, optical images of the PI/FCNT(EPD) fabric, and the final encapsulated PI/FCNT(EPD) pressure sensor. J-L, Top-view SEM images of PI/FCNT(EPD) fabric.

\section{Figure 2}

Characterization of CNT, FCNT, PI, and PI/FCNT(EPD). A-C, TEM images of CNTs. D-F, TEM images of FCNTs. G, Representative Raman spectra of CNT and FCNT. H, FT-IR spectra of CNT and FCNT.I, Representative Raman spectra of PI, FCNT, and PI/FCNT(EPD). J, XPS survey (C1s) spectra of PI and $\mathrm{PI} / \mathrm{FCNT}$ (EPD). K, FT-IR spectra of PI and PI/CNT (EPD). L, Thermogravimetric analysis curves of PI and $\mathrm{PI} / \mathrm{FCNT}$ (EPD).

\section{Figure 3}

Finite element analysis of proposed pressure sensors. A-C, Microscopic deformation process for the model with PI/CNT(Stirring), PI/FCNT(Stirring), and PI/FCNT(EPD), respectively. D, Contacting area as a function of time for the proposed four sets of pressure sensors. E, Contacting area vs. Pressure curve for $\mathrm{PI} / \mathrm{FCNT}$ (EPD) pressure sensor.

\section{Figure 4}

Pressure sensing performance of the fabricated pressure sensors. A, Relative current change vs. the pressure applied to the PI/CNT(EPD), PI/CNT(Stirring), PI/FCNT(Stirring), and PI/FCNT(EPD) pressure sensor. B, Relative current change vs the pressure applied to the PI/FCNT(EPD) pressure sensor. Inset: Plots of current variation versus pressures up to $3.38 \mathrm{MPa}$ and the corresponding sensitivity. C, Equivalent circuit model of the PI/FCNT(EPD) piezoresistive pressure sensor. D, Schematic illustration for the contacting mode between the PI/FCNT(EPD) fibers during the compression process. E-F, Relative current change vs the pressure applied to PI/FCNT(EPD) pressure sensor during the rapid and slow densification region, respectively. G, I-V curves of the PI/FCNT(EPD) sensor device under different applied pressures. $\mathrm{H}$, Multiple cycles of pressure response under different pressures ranging from $1.1 \mathrm{MPa}$ to 7.2 MPa. I, The durability test for the PI/FCNT(EPD) pressure sensor. J, Response and relaxation time of the $\mathrm{PI} / \mathrm{FCNT}$ (EPD) pressure sensor. $\mathrm{K}, \mathrm{I}-\mathrm{V}$ curves with different loading pressure ranging from $0 \mathrm{~Pa}$ to $5 \mathrm{MPa}$ 
at $100{ }^{\circ} \mathrm{C}$. L, The relative current variation of the PI/FCNT(EPD) pressure sensor under repeated pressures ranging from $250 \mathrm{KPa}$ to $4.5 \mathrm{MPa}$ at $100^{\circ} \mathrm{C}$.

\section{Figure 5}

Application of the PI/FCNT(EPD) pressure sensor. A-B, Detection of faint pressure: optical image and current curve of the proposed PI/FCNT(EPD) pressure sensor pressed by a small meter screw $(0.161 \mathrm{~g}$, $\sim 63.1 \mathrm{~Pa}$ ); Current curves of the proposed PI/FCNT (EPD) pressure sensor pressed by an aluminum particle with $0.021 \mathrm{~g}$ and $0.053 \mathrm{~g}(0.021 \mathrm{~g}+0.032 \mathrm{~g})$, respectively. Faint pressures ( 8.2 Pa) can be detected by the PI/FCNT(EPD) pressure sensors. C-F, Detection of low pressure: monitoring finger bending, wrist movement, elbow bending, and ankle movement. G, A wireless, real-time pressure monitoring system, including PI/FCNT(EPD) pressure sensor, processing unit, and mobile APP. Right of G: detection of relatively low pressure under high pressure. $\mathrm{H}-\mathrm{I}$, Detection of high pressure: Real-time pressure monitoring of the sensor device when subjected to high pressure applied by driving a car over the sensor device repeatedly. J-L, Intelligent robots 'feel' the pressure under a high-temperature environment: IR images of the mechanical hand with the PI/FCNT(EPD) pressure sensor as the mechanical hand get close to and touches the hot object $(\mathrm{J}, \mathrm{K})$; Signal response of the intelligent robot (with the integrated proposed sensor as the perception layer) during the 'close, touch, feel and response' process.

a PIIFCNT(EPD) Cu electrod b

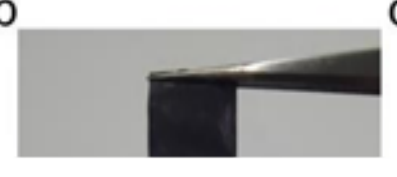

C

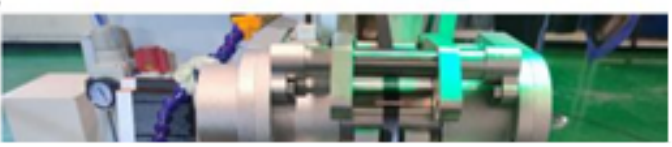




\section{Figure 6}

Application of the $\mathrm{PI} / \mathrm{FCNT}(\mathrm{EPD})$ pressure sensor in both high temperature and high pressure environment. A-B, PI/FCNT (EPD) pressure sensor, the whole structure is PEEK-Cu electrode$\mathrm{PI} / \mathrm{FCNT}$ (EPD) film-Cu electrode-PEEK. C-D, Experimental setup for the real-time detection of pre-stresses within the composite sheath of motor rotor under high temperature $(380 \mathrm{oC})$. E, Relative current change vs. the pressure applied to the PI/FCNT(EPD) pressure sensor during the winding process.

\section{Supplementary Files}

This is a list of supplementary files associated with this preprint. Click to download.

- SupplementaryMaterials.docx 